\title{
TEMPORAL ANALYSIS OF ADAPTIVE FACE RECOGNITION
}

\author{
Zahid Akhtar ${ }^{1}$, Ajita Rattani ${ }^{2}$ and Gian Luca Foresti ${ }^{1}$ \\ ${ }^{1}$ Dept. of Mathematics and Computer Science, University of Udine, Italy. \\ \{zahid.akhtar,gianluca.foresti\}@uniud.it \\ ${ }^{2}$ Dept. of Computer Science and Electrical Engineering, University of Missouri at Kansas City, USA. \\ rattania@umkc.edu
}

\begin{abstract}
Aging has profound effects on facial biometrics as it causes change in shape and texture. However, aging remains an under-studied problem in comparison to facial variations due to pose, illumination and expression changes. A commonly adopted solution in the state-of-the-art is the virtual template synthesis for aging and de-aging transformations involving complex 3D modelling techniques. These methods are also prone to estimation errors in the synthesis. Another viable solution is to continuously adapt the template to the temporal variation (ageing) of the query data. Though efficacy of template update procedures has been proven for expression, lightning and pose variations, the use of template update for facial aging has not received much attention so far. Therefore, this paper first analyzes the performance of existing baseline facial representations, based on local features, under ageing effect then investigates the use of template update procedures for temporal variance due to the facial age progression process. Experimental results on FGNET and MORPH aging database using commercial VeriLook face recognition engine demonstrate that continuous template updating is an effective and simple way to adapt to variations due to the aging process.
\end{abstract}

\section{Introduction}

Facial biometrics is one of the most acceptable biometrics as it is the commonly adopted methods of identification in human visual interaction. It is a non-intrusive way and can be used with existing image capture devices (webcams, security cameras etc.). Different vendors use different methods for face recognition, however, all of them focus on the measures of key and distinguishing features of the face. As a person's face can be captured by a camera from some distance away, facial recognition has a covert or surveillance (CCTV) capability. For these reasons, face is the commonly used biometrics in various biometric identity management programs such as US-VISIT, UIDAI and National ID cards and consumer ID etc. However, with the rapid gain in the use and popularity of facial biometrics, there also exist a need of more robust, reliable and accurate face recognition system [1, 2, 3, 7, 6, 42].

A facial biometric verification system consists of two main processes; enrollment and verification. In enrollment, individual's face samples are captured, pre-processed and features are then extracted. These extracted features are labelled with user's ID called the "template", representing his identity. Verification mode verifies claimed identity by comparing input sample(s) to the enrolled template(s). The efficiency of the facial biometric systems depends on the representative capability of the enrolled templates. Performance of these systems can be mea- 


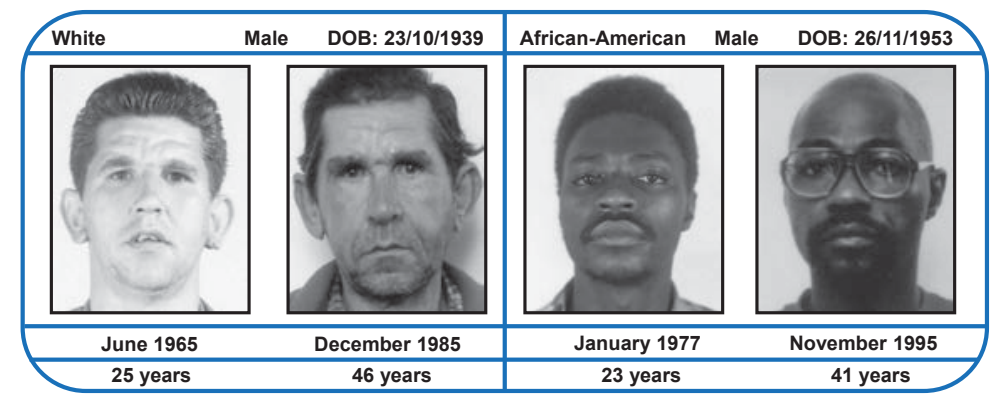

Figure 1. Example of facial images containing variations like facial hair, glasses and illumination apart from the ageing effect.

sured in terms of false acceptance rate (FAR) and false rejection rate (FRR).

According to the latest report by Facial Recognition Vendor Test (FRVT) 2013 [7], there has been improvement in the order of magnitude under controlled environment since FRVT 2006. However, system implementation in uncontrolled environment, causing variations (for instance pose, illumination and expression) in the input data, still results in performance degradation. Apart from these, facial biometrics is also subject to aging which is a progressive accumulation of changes with time and how fast we age varies from one person to another and is usually affected by our genes, environmental influences, life style and effects both the shape and the texture. Figure 1 shows the example of facial images with variates such as pose, illumination changes and facial hairs apart from the ageing effect.

An important consequence of the aging process is that enrolled templates become unrepresentative of the input (query) data after a certain time lapse as a result of change in the data distribution of an individual. This establishes its similarity with the concept drift theory [8], dealing with changing concept (models) over time and offering variant updating procedures as the solution. References $[9,10,11,12]$ report evidences of performance degradation as a result of time lapse between enrolled and query data for facial biometrics. However, facial aging has not received substantial attention in comparison to other facial variations.

Commonly adopted solution as proposed in the state-of-the-art literature is the virtual biometric synthesis of aging and de-aging transformation based on the simulation of the craniofacial morphological changes $[14,15,16,17]$ involving com- plex 3D modelling techniques. FGNET [18], MORPH [19] and BROWNS [20] are commonly adopted databases for the evaluation of these methods. However, performance gain obtained due to these methods may be limited as,

- Aging is a complex process which differs from person to person and occurs in different manifestation in different age groups.

- The simulation process is affected by other variations such as facial hairs, glasses, pose, lightning and expression, as present in the database.

These factors may cause the age transformation procedure to be prone to estimation errors (estimating growth parameters) in simulating the aging effect. In [15], on applying the age transformation on real face images, it was observed that the prediction is good for small age transformations and poor for large age transformation.

Another solution quite feasible is to continuously adapt enrolled templates to the variation of input data $[21,22,23]$. Recently template update procedure have received significant boost in biometric community and their efficiency has been proved on evaluation of the resulting performance gain of the system [21, 22, 41]. These methods may be adopted for template improvement or to avoid template aging.

- Template improvement: To increase the representational capability of the enrolled templates by appending new features and samples available during the online operation. Template improvement also includes adaptation to variations like illumination, sensor and other environmen- 
tal changes causing mismatch conditions due to unrepresentative enrolled templates.

- Template aging: To adapt the templates to the permanent changes of the input data due to the aging process over time.

Update procedures have been used in template improvement for adaptation to facial variations like expression, lightning, changing sensors and acquisition environment etc. [24, 25, 26, 27, 28]. On the other hand, template updating can offer an effective solution to aging problem as well, for many real time applications like online banking, ATM etc., where the interactive of the user is involved and expected after at least certain maximum time period.

However, to the best of our knowledge, in contrast to template improvement, template aging have not received much attention from adaptation viewpoint. In addition, there exist no study for the comparative performance evaluation of the existing face recognition systems under the ageing effect (temporal variance). This evaluation is important because it will allow to gauge the performance gain of the face recognition systems on employing ageing invariant solutions and facilitate the designer/researcher in choosing the most robust face recognizer to be integrated with ageing invariant solutions for optimal performance. Thus, this paper, which is based on our preliminary work [13], aims to:

- Evaluate and compare existing face recognition systems based on six different facial representations under the ageing effect.

- Introduce template aging as a concept drift problem.

- Evaluate the effectiveness of update procedures on facial template aging for the real time user interactive scenarios.

Experimental results on FGNET and MORPH aging database show that template updating can result in significant performance gain of the system undergoing aging for many biometric applications where the interaction of the user is expected after certain maximum time gap.

The rest of the the paper is organized as follows. Section 2 discusses how face goes under aging. Section 3 gives technical detail on template updating in terms of attributes, learning methodologies adopted and the similarity between template aging and concept drift. Experimental evaluations and results are reported in section 4. Conclusion and future work is given in section 5 .

\section{Facial Age Progression in Human}

Face ageing in humans is the result of a multidimensional process of physical, psychological, and social change, which affects considerably the appearance of a human face. Ageing related appearance variation due to bone growth normally occurs throughout childhood and puberty whereas skin related effects principally appear in older subjects. From a computer vision perspective, the challenging problem of facial aging can be described as follows:

1 Diversity of Aging Variation: The rate of aging vary from person to person. Moreover, during different age stages the facial aging effects take different forms. In addition, anti-aging products or cosmetic surgeries can also be used to deliberately intercede with the aging process. Therefore, common aging patterns or models might not be applied successfully to all subjects.

2 Shape and Texture: Generally, facial aging causes both change in shape and texture of the human face. Therefore, facial aging may be modelled by attributing facial shape and facial texture as functions of time.

3 Factors: Ideally, facial aging effects are induced by multiple factors, namely intrinsic and extrinsic factors. Biological elements causing aging are known as intrinsic factors whereas environmental influences are called extrinsic factors.

4 Controllability: Under normal mode the effects of aging can not be controlled and/or reversed. Besides, the process of collecting training data suitable for studying the effects of aging requires long time intervals.

5 Feature Selection: One of the important step of modelling facial ageing or age-invariant face recognition method is identifying the appropriate features that provide a fair description of the process. 


\section{Template Update and Age Pro- gression}

A typical biometric system is trained with the limited training data and under static environmental conditions in mind. However, the real time deployment suffers from performance loss as the enrolled templates become unrepresentative or outdated to the changing environment and variations in the input data. Novel solution to this problem is in the form of template update procedures that continuously adapt enrolled templates to the variation of the input data available during the online operation of the system. Different techniques may be adopted in learning the variations of the input data. Thus, using continuous updating, the performance loss owing to outdated templates may be avoided. Based on the adopted learning methodology, input data is used to adapt the enrolled templates. These template updating procedure can be distinguished on the basis of the following attributes [21, 22]:

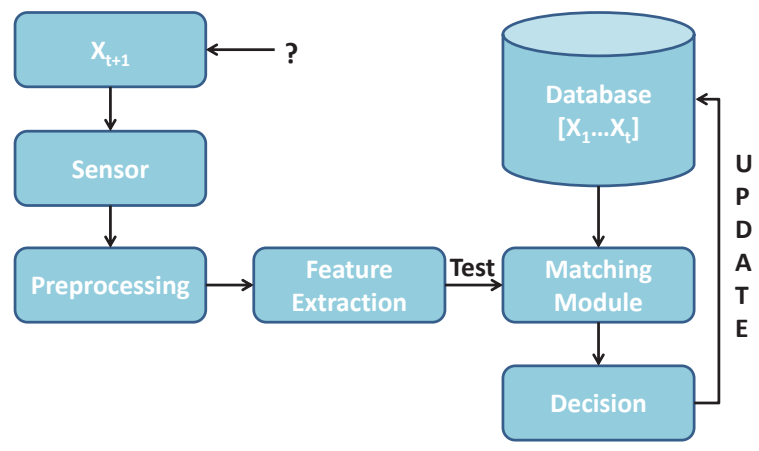

Figure 2. An example of online learning in which the input sample $X_{t+1}$ is used to update the current template $\left(X_{1}, \cdot, X_{t}\right)$ based on some learning methodology.

- Learning methodology adopted: For instance, self-training, in which the classifier or the system update itself or co-training, where two complementary biometrics update each other to the variation of the input data. Detailed information on various learning methodologies can be found in [22].

- Online vs Offline: In online method, the system is updated as soon as the input data is available, while in offline method, after a batch of data has been collected. Online systems are order sensitive in which sequence of instances are observed, one instance at a time, not necessarily in equally spaced time intervals. Let $\left(x_{1}, \cdots, x_{t}\right)$ be current template(s) at time $t$ and instance $x_{t+1}$ is the test instance used to update the model. A diagrammatic description of the online learning algorithm is given in the Fig. 2.

- Supervised or unsupervised: For supervised methods, input data is labelled by the human supervisor in an offline manner. For unsupervised methods, the input data is labelled by the classifier itself. The positively labelled data, either in supervised or unsupervised mode, is used for adapting the enrolled templates.

- Template management: These systems can also be distinguished on the basis of memory buffer utilized and how the updated template sets are maintained.

- Appending based: New features/samples are appended to the feature/template set of the old template(s), keeping full memory intact.

- Replacement based: New template is replaced with the old template. They are no memory based methods.

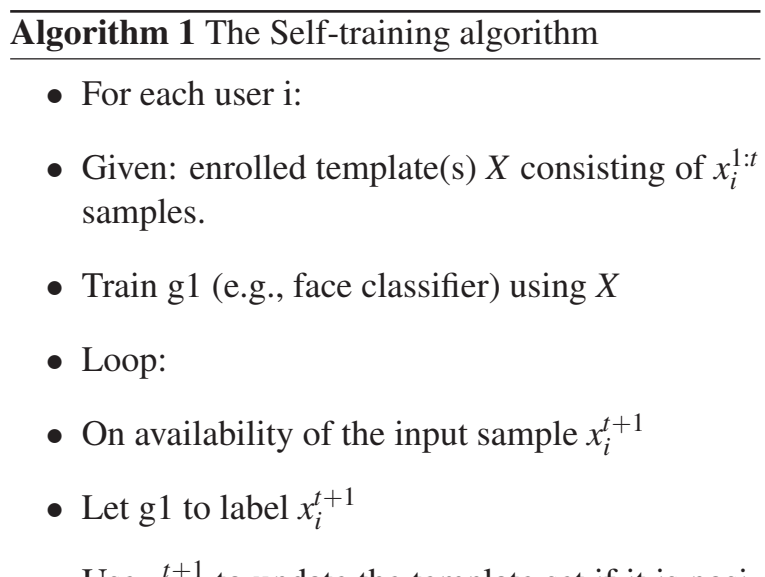

- Use $x_{i}^{t+1}$ to update the template set if it is positively classified with high confidence. (both appending where $X=x_{i}^{1: t+1}$ or replacement based $X=x_{i}^{t+1}$ management schemes can be used in updating).

- Retrain g1 using $X$

The most commonly adopted learning methodology in the state-of-the-art literature [22, 24, 29, 30] is self-training [21] based learning in which the classifier adapts itself to the variation of the input data. These methods consist of two basic steps a) 
Table 1. Database characteristics of the state-of-the-art methods for update procedures.

\begin{tabular}{|c||c||c|}
\hline Reference & Database & Variations \\
\hline$[24]$ & Homemade & Expression, Pose and Illumination \\
\hline$[25]$ & GEFA & Expression, Illumination, Pose and Uncontrolled background \\
\hline$[26]$ & Equinox & Illumination \\
\hline$[27]$ & BANCA & Expression, Illumination and Uncontrolled background \\
\hline$[28]$ & Big brother & Pose and Illumination \\
\hline$[32]$ & AR & Expression, Occlusion and Illumination \\
\hline
\end{tabular}

label assignment to the input data and, b) adaptation. Typically, these are continuous incremental learning technique in which the positively classified (with high confidence) input sample is used to update the template gallery. Both the appending or replacement based template management strategies can be adopted for updating. These are unsupervised user-specific process i,e., adopted independently for each user [24, 29, 30].

Certain, supervised methods have also been introduced in the literature in which the human supervisor labels the input data [27,31], which is then used for adaptation. They are offline methods and the updating process is carried after certain fixed time period. Information on other learning methodologies like co-training, graph based methods can be found in [21] and [22].

The detailed description of the self-training is given in the algorithm 3. For supervised methods, the algorithm will be same expect that instead of classifier assigning label to the input data, a human supervisor assigns labels.

As mentioned before, updating mechanism has been commonly adopted for template improvement and has reported significant performance gain using different learning methodologies. Table 1 refers to the database characteristics of the state-of-theart procedures for updating for facial biometrics. The database characteristics contain variations related to mismatch conditions, i.e., change in illumination, sensor, occlusion, pose, expression etc. Reference [25] evaluates the template-updating effect on GEFA (Gradual evolution of facial appearance) but the database is acquired over a period of five months which fails to capture aging variations. Referred works in Table 1:

- shows significant performance gain on the adoption of update procedures.
- Commonly adopted learning technique is selftraining.

\subsection{Template aging, updating and concept drift}

In comparison to other facial variations (pose, illumination, etc.), adaptation to template aging deserves a dedicated treatment of its own as:

- Aging is a life long process.

- Brings gradual changes in the data-distribution over time, thus causing performance loss as a result of template becoming outdated.

- Order sensitive adaptation is needed, i.e., template is adapted using the input samples in the order of its availability.

These factors indicate that template aging process is very similar to the concept drift theory [8], based on the fact that real world concepts change with time resulting in,

- underlying data distribution to change, and

- changes in data distribution may be incremental or decremental. Namely, these changes may show increasing or decreasing trends.

Thus, causing the model learned on the old concept inconsistent with the new data. One of the efficient solutions as offered for the concept drift problem is regular updating.

\section{Experiments}

In this section, we provide experimental evaluation of the performance of facial recognition methods under ageing effects, template ageing as con- 
cept drift problem and template update procedures on facial ageing.

\subsection{Performance of Face Recognition Sys- tems under Ageing}

Facial Representations: In this study, following six facial representations were considered: Local Binary Pattern (LBP) [34], Multi-scale Local Binary Patterns (MLBP) [35], LPQ (Local Phase Quantization) [36], LTP (Local Ternary Patterns) [37], EBGM (Elastic Bunch Graph Matching) [38], SIFT (Scale Invariant Feature Transform) [39] and SURF (Speeded Up Robust Features) [40].

Briefly, Local Binary Pattern (LBP) operator forms labels for the image pixels by thresholding the neighborhood of each pixel with the center value and considering the result as a binary number [34]. MLBP is an extended version of LBP by using multiple radii and offering the advantage of scale invariance [35]. Local Phase Quantization (LPQ) utilizes phase information computed locally in a window. The phases of the four low-frequency coefficients are decorrelated and uniformly quantized [36]. In LTP (Local Ternary Patterns) [37], the binary code in LBP are replaced by the ternary code using central pixel value. EBGM (Elastic Bunch Graph Matching) [38] localizes a set of landmark features and extracts Gabor jets at landmark positions. SIFT (Scale Invariant Feature Transform) features are efficiently detected through a staged filtering approach and are highly distinctive.[39]. SURF (Speeded Up Robust Features) relies on integral images for image convolutions (using a Hessian matrix-based measure for the detector, and a distribution-based descriptor) [40].

Data set: We used MORPH [19] data set that comprises thousands of facial images of individuals across time, collected in real-world conditions (not a controlled collection). This dataset also include essential meta-data, such as age, sex, race, glasses, facial hair, etc. A subset of 631 subjects from MORPH (1700 images) with about 3 images per subject are used in this study. Age range of the subjects are $[15,68]$. The characteristics and image samples of MORPH dataset are shown in Table 3 and Fig. 3, respectively.
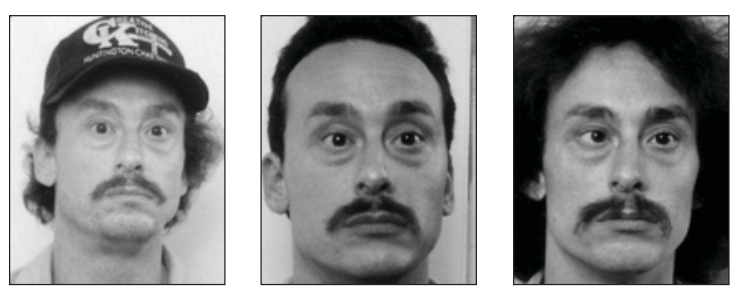

Figure 3. Sample images from MORPH databases for a user at 27, 31 and 36th year of age.

Protocol: First of all, face images are cropped to extract the face portion using the eye locations, retrieved through Meta-data file. These cropped images are first resized to $250 \times 200$ pixels. Then, the cropped images are illumination normalized by incorporating a series of steps [37] as follows 1) gamma correction, 2) difference of gaussian (DoG) filtering, 3) masking, and 4)contrast equalization. This preprocessing chain combat the effect of illumination, local shadowing and highlights. While still preserving the essential elements of visual appearance of use in recognition. Readers are referred to [37] for the detailed information on the preprocessing steps for illumination normalization.

Then, facial features are extracted from all images in the database using the considered facial representations. Next, the following steps are performed for performance evaluation:

- Similarity(dissimilarity) matrix is computed using all-pair matching of facial features. Matching scores are divided into an authentic (genuine) and an impostor score distribution.

- The data set (matching scores) is bootstrapped at the user level, i.e., subset of users are selected with replacement for performance evaluation.

- Performance has been evaluated on calculating area under curve (AUC) statistic on the bootstrapped data set. AUC is computed as a function of true accept rate (TAR) and false accept rate (FAR) as :

$$
A U C=\int_{0}^{1} T A R(F A R) d F A R
$$

The AUC value ranges from 0 to 1 .

- Variation in AUC on the bootstrapped data set is recorded as mean \pm std. 


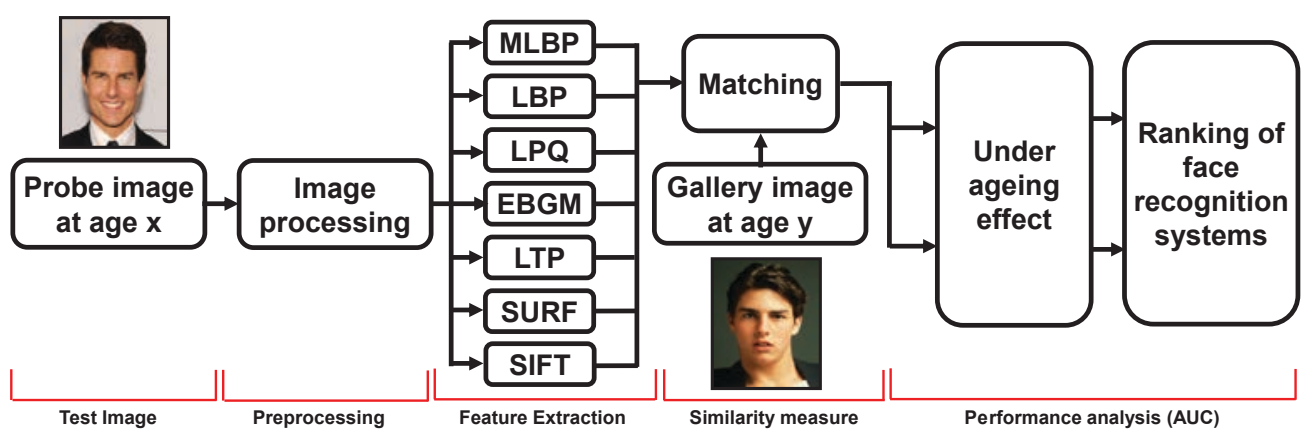

Figure 4. Schematic of the procedure of performance evaluation for the face recognition systems under ageing effect.

- Finally, face recognition systems are ranked in the descending order on the basis of their average AUC on the bootstrapped data set. Figure 4 illustrates the overall schematic of the procedure for performance evaluation of face recognition systems under ageing effect.

Table 2. AUC values obtained on the performance evaluation of six facial representations under the ageing effect on the MORPH database.

\begin{tabular}{|c|c|c|c|}
\hline Reference & $\begin{array}{c}\text { Face } \\
\text { representation }\end{array}$ & mean \pm std & $\begin{array}{c}\text { Percentiles } \\
{[25 \%, 50 \%, 75 \%]}\end{array}$ \\
\hline$[35]$ & MLBP & $0.66 \pm 0.02$ & {$[0.58,0.60,0.64]$} \\
{$[34]$} & LBP & $0.64 \pm 0.08$ & {$[0.56,0.59,0.63]$} \\
{$[36]$} & LPQ & $0.62 \pm 0.01$ & {$[0.59,0.60,0.61]$} \\
{$[38]$} & EBGM & $0.60 \pm 0.02$ & {$[0.56,0.57,0.59]$} \\
{$[37]$} & LTP & $0.55 \pm 0.00$ & {$[0.50,0.51,0.54]$} \\
{$[40]$} & SURF & $0.52 \pm 0.01$ & {$[0.51,0.52,0.53]$} \\
{$[39]$} & SIFT & $0.51 \pm 0.08$ & {$[0.50,0.51,0.51]$} \\
\hline
\end{tabular}

Results: Table 2 quotes the AUC values as mean \pm std and percentile statistics on the bootstrapped dataset for all the facial representations. These facial representations are mentioned in the descending order on the basis of their AUC values.

It can be seen that all the systems resulted in low performance on the MORPH facial ageing database. MLBP based facial representation outperformed other facial representations under the ageing effect. These results indicate that MLBP is able to, to some extent, locate discriminative information even under the presence of profound facial ageing between the pair of images. Nevertheless, it could be interesting to integrate MLBP based facial recognition system with age-invariant solutions and gauge the improvement over the baseline performance, as here evaluated to be 0.66 (recorded as AUC) in Table 2.

\subsection{Template Aging as a Concept Drift Problem}

In this section, we carried out detailed analysis of template aging as a problem of concept drift.

Data set: Besides MORPH database, we also used FGNET [18] aging database containing facial images of number of subjects at different ages. This database has been generated as a part of the European Union project FGNET (Face and Gesture Recognition Research Network).

The characteristics of MORPH and FGNET databases can be found in Table 3. Apart from aging variations, these databases also offer changes in illumination, poses, expression, beard and mustaches, spectacles, hats etc., thus offering a challenging situation. Fig. 5 shows image samples of an individual from FGNET aging database.

Table 3. The characteristics of the FGNET and MORPH databases as used in the experiments.

\begin{tabular}{|c|c|c|}
\hline Characteristics & FGNET & MORPH \\
\hline No. of Subjects & 82 & 631 \\
\hline Average no. of images per subject & $6-18$ & 3 \\
\hline Age Range & $0-69$ & $15-68$ \\
\hline \multicolumn{3}{|c|}{ Other Intra-class variations } \\
\hline Illumination & Yes & Yes \\
\hline Poses & Yes & Yes \\
\hline Expression & Yes & Yes \\
\hline Beards and Moustaches & Yes & Yes \\
\hline Spectacles & Yes & Yes \\
\hline Hats & Yes & Yes \\
\hline
\end{tabular}

Protocol: Using FGNET dataset, on the basis of the age of the samples, we followed the notation that each user $i$ consisting of $x_{i}^{1: T}$ genuine samples in ascending order, i.e., age of $x_{i}^{t}$ is lesser than all the other $x_{i}^{t+1}$ samples. We analyzed the genuine 

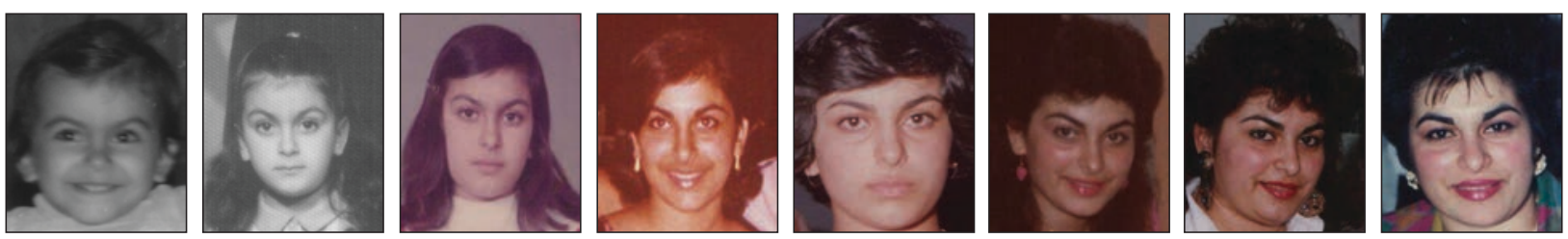

Figure 5. Sample images from FGNET database for a user at different years (3-41).

pdfs (probability distribution function) of a randomly chosen two users for the FGNET database by partitioning the samples into two and three age groups as shown in Fig. 6. In Fig. 6-Top, the pdf of the first group is obtained by computing the scores within the group and the pdf of the second group is obtained by computing matching scores via comparing the samples of the first group to that of the second group. In Fig. 6-Bottom, shows the genuine pdfs drawn by partitioning the facial samples into three groups on the basis of age and computing the genuine similarity scores for each group individually by comparing each image to all the other images within the group.
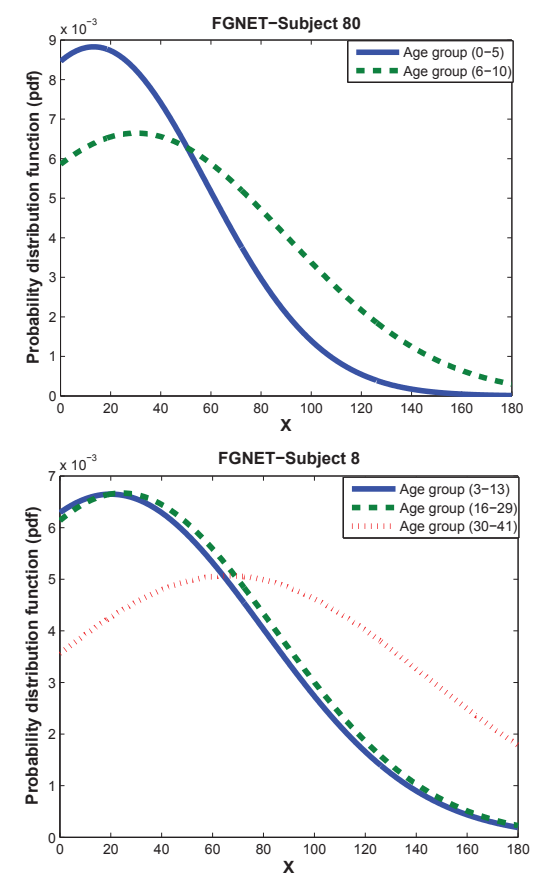

Figure 6. The probability distribution functions (pdfs) drawn by partitioning the image samples into age groups and computing the genuine score distributions.

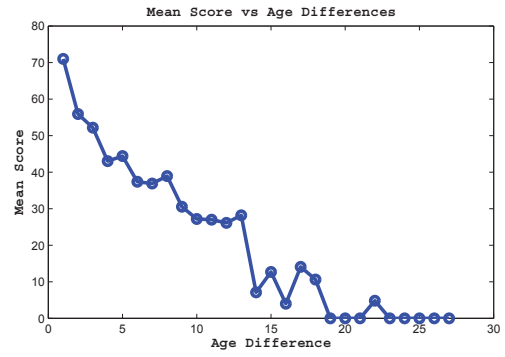

Figure 7. Scores clustered on the basis of age differences between two samples. Mean score for each cluster vs the age difference shows that the presence of decremental drift in the

data-distribution as a result of time lapse.

Further, using MORPH database, for each user $i$, the genuine scores are computed by comparing each sample $x_{i}^{j}$ to all the other $x_{i}^{k}$ samples, such that age of $x_{i}^{j}$ is lesser than all the other $x_{i}^{k}$ samples. This same process is repeated for all the $N$ users in the database. Then, all the obtained genuine scores are clustered on the basis of age difference between the two samples used for score computation, irrespective of the user. The clustered scores are then averaged (mean) and mean score vs the age difference is plotted as shown in Fig. 7. The $x$ axis in Fig. 7 shows the specific age difference and $y$ axis shows the mean of all the genuine scores belonging to samples with a specific age difference.

Results: Fig. 6 shows that the data distribution changes over time as a result of aging process. While, Fig. 7 indicates that drift is decremental. The drift is called decremental because the mean of the scores shows decreasing trend as the age differences among the samples increases. Moreover, Fig. 7 points out that template update can be an effective technique provided that the new sample is available within certain gap otherwise the updated template will again be outdated to the new incoming samples. 


\subsection{Evaluation of Template Update Proce- dures on Facial Age Progression}

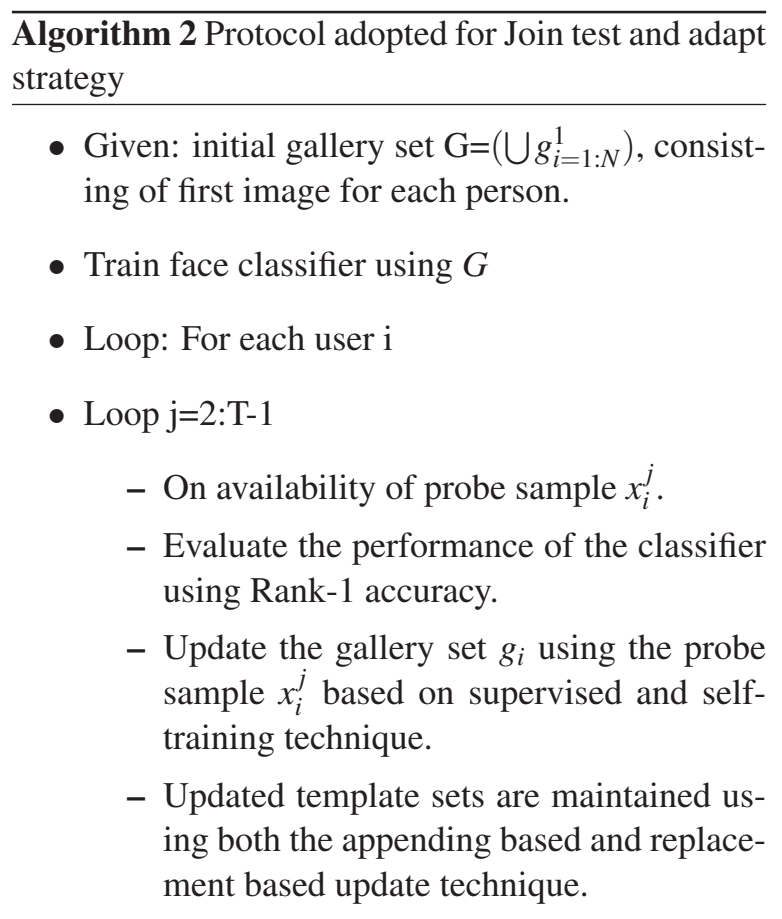

In this section, we perform experimental evaluation of template update for facial aging. Our experiments used online self-training based learning technique with supervised and unsupervised label assignment using both, the appending and replacement based template management strategies. In similar manner, other learning methodologies can be efficiently exploited as well.

Protocol: The effectiveness of the template update for the aging process can be evaluated using

- how much performance gain is expected?

- the maximum time limit before which the new sample is required for the update procedure to be effective?
Table 4. (\%) Rank-1 accuracy and performance gain of the system that keeps updating in comparison to baseline classifier that does not update. Both the supervised (for appending and replacement based scheme for managing)and self-training (with appending based management) learning methodologies are evaluated.

\begin{tabular}{|c||c||c||c||c|}
\hline Database & $\begin{array}{c}\text { Baseline } \\
\text { System } \\
\text { (Rank-1 }(\%) \\
\text { accuracy) }\end{array}$ & $\begin{array}{c}\text { Appending } \\
\text { (supervised) }\end{array}$ & $\begin{array}{c}\text { Replacement } \\
\text { (supervised) }\end{array}$ & $\begin{array}{c}\text { Appending } \\
\text { (unsupervised) }\end{array}$ \\
\hline FGNET & 12.79 & 70.09 & 68.68 & 29.70 \\
\hline MORPH & 66.62 & 88.83 & 82.46 & 82.59 \\
\hline
\end{tabular}

Online (incremental) learning updates the system as soon as the input sample is available. For the evaluation of these systems Join test and adapt strategy has been commonly adopted in the literature [22], in which the available input sample is first used to evaluate the performance of the updated system, followed by update [24, 30, 32]. We have also adopted join test and adapt based update and performance evaluation due to its efficiency in better utilizing the limited set of available samples. The complete protocol for online learning together with performance evaluation is given in Algorithm 2 , where $\mathrm{T}$ is the maximum no. of instances available in the database.

Commercial VeriLook software [33] face identification engine has been used for experimental analysis which consist of two basic modules enrollment and identification. Based on the face identification terminology, during enrollment face image is captured, aligned, face detected and feature sets are extracted and template formed, representing the gallery $g_{i}$ of a user $i$. The gallery set $G$ consist of all the gallery images of $N$ subjects in the database, i.e, $G=\cup_{i=1}^{N} g_{i}$. On identification, probe image $p$ presented to the system is matched with the gallery set $G$ and the identities are retrieved on the basis of matching score above a set threshold. Performance evaluation of the identification system is done using Rank-1 accuracy at 1\% FAR operating point, which means that $\%$ number of times correct identity is ranked first in the list of retrieved identities. 

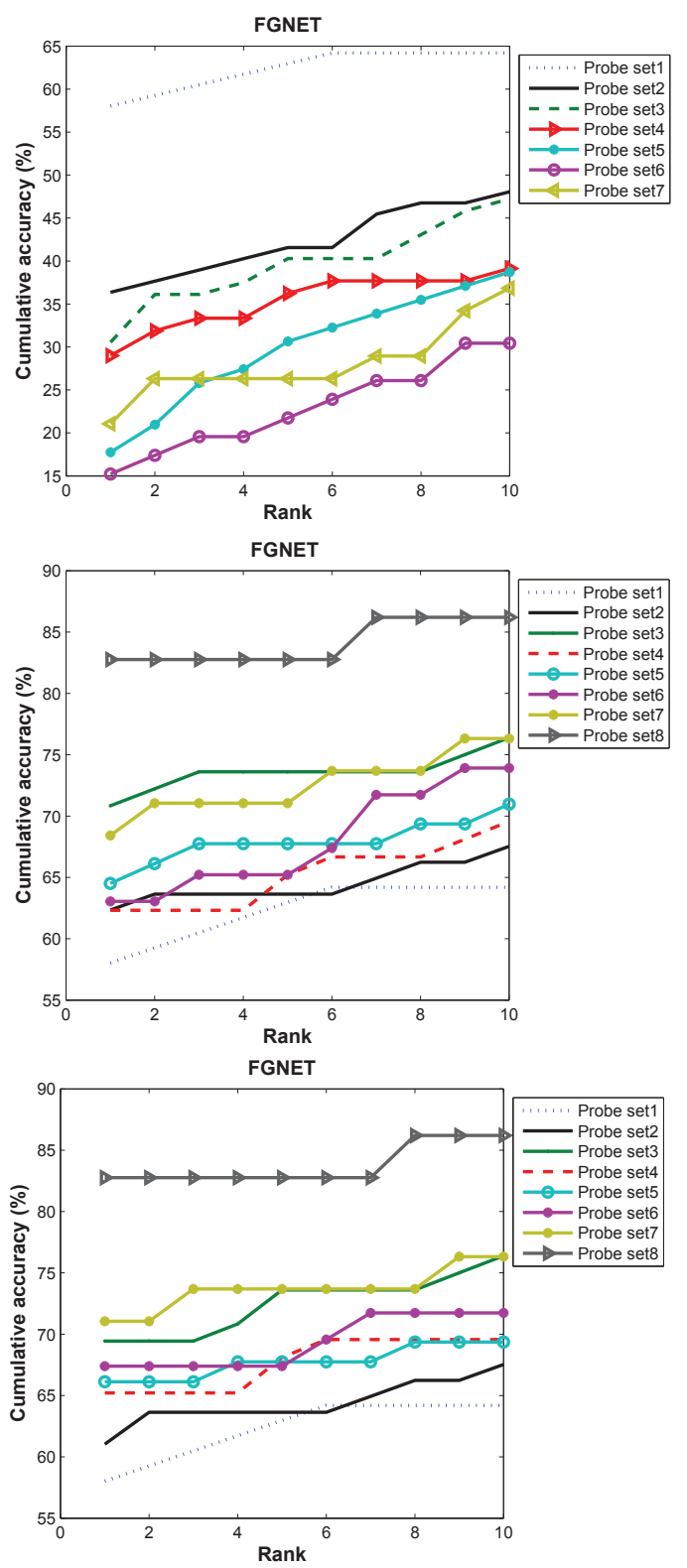

Figure 8. Top: CMC Curves for semi-supervised appending based learning for FGNET Database with threshold set at $1 \%$ FAR. Middle: CMC

Curves for active replacement based online learning for FGNET Database. Bottom: CMC Curves for active appending based online learning for FGNET Database.


Figure 9. Top: CMC Curves for online active appending based learning for MORPH Database. Middle: CMC Curves for online active replacing based learning for MORPH Database. Bottom:

CMC Curves for online updating based on semi-supervised appending based learning for MORPH Database with threshold set 0.01FAR.

Results: Table 4 presents the baseline accuracy (when the first image is in the template gallery for each user) and the averaged Rank-1 accuracy of the system that continuously adapts itself. There is a remarkable increase in the performance of supervised labelling based self-training for both appending and replacement based template management schemes. However, for unsupervised label assignment (selftraining) using appending based technique, FGNET 
does not show much improvement due to the challenging nature of the dataset, thus resulting in very low genuine scores. It can also be seen that appending based supervised labeling is better than replacement based update, i.e., retention of prior knowledge (or old templates) is worthwhile and help to attain more performance gain. Hence, template update schemes can result in substantial performance gain of the system under aging.

Figures 8 and 9 show Cumulative Match Characteristic (CMC) Curves for active (appending, replacing based template management strategies) and semi-supervised (appending based) learning technique for the FGNET and MORPH databases, respectively, for each probe set.

As mentioned before, another vital question is what is the maximum time limit before which the new sample is required for the update procedure to be effective?. Accordingly, the difference in the averaged Rank-1 accuracy of the update based system with those of the baseline for the users grouped on the basis of averaged age difference among their samples is computed for MORPH database using appending based supervised labelling technique as given in Table 5. The results obtained with FGNET database were qualitatively very similar.

Though no specific trend can be noticed in the performance gain in respect to averaged age difference among the samples. But, it can be seen that till the averaged age difference of nine years, the gain due to update is in double figures then from 10 to 14 years, where gain degrades sharply. It can also be noticed that after 14 years, there is no gain in the performance due to the update procedure. This observation depicts that the maximum time gap in the availability of input sample for the effective update procedure is 9 years according to the Table 5 . This figure will change with the database, acquisition set-up and the classifier used. Nevertheless, experimental results prove the efficacy of the update procedures for the variations due to template aging.

\begin{tabular}{|c|c|c|c|}
\hline $\begin{array}{c}\text { Age } \\
\text { difference }\end{array}$ & $\begin{array}{l}\text { \# No. of } \\
\text { subjects }\end{array}$ & $\begin{array}{l}\text { Performance gain } \\
\text { (offline) supervised } \\
\text { (appending) }\end{array}$ & $\begin{array}{c}\text { Performance gain } \\
\text { (online) unsupervised } \\
\text { (appending) }\end{array}$ \\
\hline 1 & 78 & 13.02 & 5.5 \\
\hline 2 & 89 & 10 & 9.14 \\
\hline 3 & 63 & 15.74 & 3.6 \\
\hline 4 & 49 & 11.93 & 7.4 \\
\hline 5 & 56 & 26 & 1.97 \\
\hline 6 & 48 & 20 & 13.52 \\
\hline 7 & 29 & 11.59 & 3.50 \\
\hline 8 & 22 & 13.89 & 4.16 \\
\hline 9 & 22 & 27.38 & 0 \\
\hline 10 & 13 & 4.54 & 0 \\
\hline 11 & 15 & 1.78 & 0 \\
\hline 12 & 10 & 0 & 0 \\
\hline 13 & 8 & 6.25 & 0 \\
\hline 14 & 6 & 8.3 & 0 \\
\hline 15 & 4 & 0 & 0 \\
\hline 16 & 7 & 0 & 0 \\
\hline 17 & 4 & 0 & 0 \\
\hline 18 & 4 & 0 & 0 \\
\hline 19 & 1 & 0 & 0 \\
\hline 21 & 2 & 0 & 0 \\
\hline 22 & 2 & 0 & 0 \\
\hline
\end{tabular}

Table 5. Difference in the averaged Rank-1 accuracy of the update based system with those of the baseline for the users grouped on the basis of averaged age difference among their samples.

\section{Conclusions}

In the long term, the performance of a face recognition system is affected by the facial age progression, which leads to significant alterations in the human faces. Therefore, in this study first six baseline facial representations based on local features were evaluated under facial ageing impact, to show that how severely aging degrades the performances. Performance loss due to facial age progression is the result of change in the datadistribution causing the templates to be outdated over time, making thus the process similar to the concept drift theory. Recently update procedures have been introduced offering effective and simple way for the template adaptation. However, effectiveness of these methods for aging variations have not been evaluated till date. Experimental results, in the previous section, on commonly used facial aging databases (FGNET and MORPH) ensures that template update can effectively adapt the system to temporal variance. Future works will focus on

- Updating only on change detection, in which the system updates itself only if the input sample exhibit changes related to aging. 
- Employing gradual time based forgetting mechanism in which the recent samples are given more weights in comparison to older samples, resulting in gradual forgetting which is quite efficient for template management and life long learning.

- Combination of template updating and age transformation based technique for the case when the input samples is not available within a certain time interval. Note that the age transformation process cannot be employed regularly for updating the enrolled template as it is a complex process and prone to estimation error, which may result in cumulative errors in this case.

- Tackling the impostor intrusion into the updated template set due to successful zero and non-zero effort impostor attacks.

\section{References}

[1] Zahid Akhtar, Security of Multimodal Biometric Systems against Spoof Attacks, $\mathrm{PhD}$ thesis, University of Cagliari, Italy, 2012.

[2] N. Poh, J. Kittler and A. Rattani, and M. Tistarelli, Group-specific score normalization for biometric systems, Proc. IEEE Conference on Computer Vision and Pattern Recognition Workshops (CVPRW), pp. 38-45, 2010.

[3] Z. Akhtar, S. Kale and N. Alfarid, Spoof Attacks on Multimodal Biometric Systems, Proc. Int'l Conference on Information and Network Technology (ICINT), pp. 46-51, 2011.

[4] Z. Akhtar, C. Micheloni, C. Piciarelli, G. L. Foresti, MoBio_LivDet: Mobile Biometric Liveness Detection, IEEE Int'1 Conf. on Advanced Video and Signal Based Surveillance (AVSS), pp. 187192, 2014.

[5] Z. Akhtar and N. Alfarid, Secure Learning Algorithm for Multimodal Biometric Systems against Spoof Attacks, Proc. Int'l Conference on Information and Network Technology (ICINT), pp. 52-57, 2011.

[6] Z. Akhtar, C. Micheloni and G. L. Foresti, Biometric Liveness Detection: Challenges and Open Research Opportunities, IEEE Security \& Privacy, 2015.

[7] FRVT 2013, http://www.nist.gov/itl/ iad/ig/frvt-2013.cfm/
[8] A. Tsymbal, The problem of concept drift: Definitions and related work, Department of Computer Science, Trinity College, Ireland, 2004.

[9] Z. Akhtar, A. Rattani, A. Hadid and M. Tistarelli, Face Recognition under Ageing Effect: A Comparative Analysis, Proc. Int'l Conf. on Image Analysis and Processing (ICIAP), pp. 309-318, 2013.

[10] P. J. Flynn, K. W. Bowyer and P. J. Phillips, Assessment of time dependency in face recognition: An initial study, Proc. 4th Int. Conf. on Audio and Video based Biometric Person Authentication, pp. 44-51, 2003.

[11] H. Ling, S. Soatto, N. Ramanathan and D. W. Jacobs, A study of face recognition as people age, Proc. 11th IEEE Int. Conf. on Computer Vision (ICCV), pp. 1-8, 2007.

[12] A. Rattani, B. Freni, G. L. Marcialis and F. Roli, Template Update Methods in Adaptive Biometric Systems: A Critical Review, Proc. International Conference on Biometrics (ICB), pp. 847$857,2009$.

[13] Z. Akhtar, A. Ahmed, C. E. Erdem and G. L. Foresti, Biometric Template Update under Facial Aging, IEEE Symposium on Computational Intelligence in Biometrics and Identity Management, 2014.

[14] A. Lanitis, C. J. Taylor and T. F. Cootes, Toward automatic simulation of aging effects on face images, IEEE Tran. on Pattern Analysis and Machine Intelligence, 24(4):442-455, 2002.

[15] N. Ramanathan and R. Chellappa, Face verification across age progression, Proc. IEEE Conf. Computer Vision and Pattern Recognition (CVPR), pp. 462-469, 2005.

[16] N. Ramanathan and R. Chellappa, Modeling age progression in young faces, Proc. IEEE Conf. Computer Vision and Pattern Recognition (CVPR), pp. 387-394, 2006.

[17] U. Park, Y. Tong and A. K. Jain, Age-invariant face recognition, IEEE Tran. on Pattern Analysis and Machine Intelligence, 32(5):947-954, 2010.

[18] FGNET Aging Database, http://www. fgnet.rsunit.com/

[19] K. J. Ricanek and T. Tesafaye, Morph: A longitudinal image database of normal adult ageprogression, Proc. Int'l Conf. on Automatic Face and Gesture Recognition (FG), pp. 341-345, 2006.

[20] N. Nixon and P. Galassi, The brown sisters, thirtythree years, In The Museum of Modern Art, NY, USA, 2007. 
[21] A. Rattani. Adaptive Biometric System based on Template Update Procedures, $\mathrm{PhD}$ thesis, University of Cagliari, Italy, 2010.

[22] N. Poh, A. Rattani and F. Roli, Critical Analysis of Adaptive Biometric Systems, IET Biometrics, 1(4):179-187, 2012.

[23] A. Rattani and A. Ross, Automatic Adaptation of Fingerprint Liveness Detector to New Spoof Materials, In Proc. IEEE International Joint Conference on Biometrics (IJCB), 2014.

[24] X. Liu, T. Chen, and S. M. Thornton, Eigenspace updating for non-stationary process and its application to face recognition, Pattern Recognition, pp. 1945-1959, 2003.

[25] S. K. Pavani, F. M. Sukno, C. Butakoff, X. Planes and A. F. Frangi, A confidence based update rule for self-updating human face recognition systems, Proc. Int'l Conf. on Biometrics (ICB), pp. 151-160, 2009.

[26] A. Rattani, G.L. Marcialis and F. Roli, Biometric template update using the graph mincut: a case study in face verification. Proc. 6th IEEE Biometric Symposium, 2008.

[27] N. Poh, J. Kittler, S. Marcel, D. Matrouf and J. F. Bonastre, Model and Score Adaptation for Biometric Systems: Coping With Device Interoperability and Changing Acquisition Conditions, Proc 20th Int'1 Conf. on Pattern Recognition (ICPR), pp. 1229-1232, 2010.

[28] A. Franco, D. Maio and D. Maltoni, Incremental template updating for face recognition in home environments, Pattern Recognition, 43:2891-2903, 2010.

[29] X. Jiang and W. Ser, Online fingerprint template improvement, IEEE Tran. PAMI, vol. 8, pp. 11211126, 2002.

[30] C. Ryu, K. Hakil and A. Jain, Template adaptation based fingerprint verification, Proc. Int'l Conf. on Pattern Recognition (ICPR), pp. 582-585, 2006.

[31] U. Uludag, A. Ross and A. Jain, Biometric template selection and update: a case study in fingerprints, Pattern Recognition, 37(7):1533-1542, 2004.
[32] F. Roli and G.L Marcialis, Semi-supervised pcabased face recognition using self training, Proc. Int'1 workshop on S+SSPR, 2006.

[33] Verilook: http://www.neurotechnology.com/

[34] T. Ahonen, A. Hadid and M. Pietikainen, Face description with local binary patterns: application to face recognition, IEEE Trans. on Pattern Analysis and Machine Intelligence, vol. 28, no. 12, pp. 2037-2041, 2006.

[35] Chi-Ho Chan, J. Kittler and K. Messer, Multi-scale Local Binary Pattern Histograms for Face Recognition, In ICB, pp. 809-818, 2007.

[36] T. Ahonen, E. Rahtu and V. Ojansivu and J. Heikkil, Recognition of blurred faces using local phase quantization, In Proc. Int. Conf. on Patt. Reco., pp. 8-11, 2008.

[37] X. Tan and B. Triggs, Enhanced Local Texture Feature Sets for Face Recognition under Difficult Lighting Conditions, IEEE Trans. on Image Processing, vol. 19, no. 6, pp. 1635-1650, 2010.

[38] L. Wiskott, J.M. Fellous, N. Kruger and C. Malsburg, Face recognition by elastic bunch graph matching, IEEE Trans. on PAMI, vol. 19, no. 7, pp. 775-780, 1997.

[39] D. R. Kisku, A. Rattani, E. Grosso and M. Tistarelli, Face Identification by SIFT-based Complete Graph Topology, In Proc. of 5th IEEE Int'1 Workshop on Automatic Identification Advanced Technologies, pp. 63-68, 2007.

[40] P. Dreuw, P. Steingrube and H. Hanselmann and H. Ney, SURF-Face: Face Recognition Under Viewpoint Consistency Constraints, In Proc. BMVC, pp. 1-11, 2009.

[41] A. Rattani, G. L. Marcialis, F. Roli, An Experimental Analysis of the Relationship between Biometric Template Update and the Doddington's Zoo in Face Verification, In Proc. of 14th Int'1 Conference on Image Analysis and Processing, 2009.

[42] Z. Akhtar, G. Fumera, G. L. Marcialis and F. Roli, Evaluation of Multimodal Biometric Score Fusion Rules under Spoof Attacks, 5th IAPR Int'1 Conference on Biometrics (ICB), pp. 402-407, 2012. 